\title{
EXAMPLES OF CAPACITY FOR SOME ELLIPTIC OPERATORS
}

\author{
JANG-MEI WU
}

\begin{abstract}
We study $L$-capacities for uniformly elliptic operators of nondivergence form

$$
L=\sum_{i, j} a_{i j}(x) \frac{\partial^{2}}{\partial x_{i} \partial x_{j}}+\sum_{j} a_{j}(x) \frac{\partial}{\partial x_{j}} ;
$$

and construct examples of large sets having zero $L$-capacity for some $L$, and small sets having positive $L$-capacity. The relations between ellipticity constants of the coefficients and the sizes of these sets are also considered.
\end{abstract}

A compact set $S \subseteq\{|x|<1\} \subseteq \mathbb{R}^{n}, n \geq 2$, has zero capacity for the Laplacian if and only if it is a removable set for the class of bounded subharmonic functions on $\{|x|<1\}$; equivalently, there exists a positive superharmonic function $(\not \equiv+\infty)$ on $\{|x|<1\} \backslash S$ which approaches $+\infty$ continuously on $S$.

In this note, we study $L$-capacities for uniformly elliptic operators of nondivergence form

$$
L=\sum_{i, j} a_{i j}(x) \frac{\partial^{2}}{\partial x_{i} \partial x_{j}}+\sum_{j} a_{j}(x) \frac{\partial}{\partial x_{j}}
$$

and construct examples of large sets having zero $L$-capacity for some $L$, and small sets having positive $L$-capacity. We also study the relations between ellipticity constants of the coefficients and the sizes of these sets.

When $\left(a_{i j}\right)$ are Dini continuous and $\left(a_{j}\right)$ are bounded, sets of $L$-capacity zero are precisely those of capacity zero for the Laplacian; this follows from the growth of the Green function for these operators. (See [1] and [7].) In general, there are elliptic operators $L$ with continuous coefficients for $n=2$, bounded coefficients for $n \geq 3$, for which a single point has positive $L$-capacity; again this reflects the behavior of the Green function. (See [2, 4, 5 and 12].)

For uniformly elliptic operators of divergence form, the growth of Green function near its pole is comparable to that for the Laplacian [10]. Therefore sets of capacity zero are exactly those for the Laplacian.

Let $L$ be the above operator and coefficients of $L$ be continuous in a domain $\Omega \subseteq \mathbb{R}^{n}$. We consider strong solutions of $L=0$ in $W_{\text {loc }}^{2, n}(\Omega)$ and call them $L$-solutions. The maximum principle, the existence and uniqueness of the solution to the Dirichlet problem and the Harnack principle are well known. A lower semicontinuous function $v$ is called an $L$-supersolution on $\Omega$, if for any closed ball $B \subseteq \Omega$ and any $L$-solution $u$ in $B$ with $u$ continuous on $\bar{B}$, the

Received by the editors August 23, 1989 and, in revised form, June 13, 1990.

1980 Mathematics Subject Classification (1985 Revision). Primary 31B15.

Research was partially supported by the National Science Foundation. 
inequality $v \geq u$ on $\partial B$ implies that $v \geq u$ in $B$. A function $v \in C^{2}(\Omega)$ is an $L$-supersolution if and only if $L v \leq 0$. A function $v$ is called an $L$-subsolution if $-v$ is an $L$-supersolution. (See [6, Chapter 9].)

Let $D \equiv\{|x|<1\}, \bar{x}=\left(\frac{1}{2}, 0,0, \ldots 0\right)$ and $S$ be a compact set in $\{|x| \leq$ $\left.\frac{1}{4}\right\}$. We define the $L$-capacity of $S$ as

$L$-cap $S=\inf \{v(\bar{x}): v$ is a positive $L$-supersolution on $D$ and $v \geq 1$ on $S\}$, when the coefficients of $L$ are bounded continuous in $D$. When the coefficients of $L$ are only known to be bounded continuous on $D \backslash S$, we say $L$-cap $S=0$ provided that

$$
\begin{aligned}
& \inf \{v(\bar{x}): v \text { is a positive } L \text {-supersolution on } D \backslash S, \\
& \left.\quad \text { and } \liminf _{x \rightarrow x_{0}} v(x) \geq 1 \text { at each } x_{0} \in S\right\}=0 ;
\end{aligned}
$$

otherwise we say $L$-cap $S>0$. Both definitions of $L$-capacity zero agree when the coefficients of $L$ are continuous on $D$. We note that if there exists a positive $L$-supersolution on $D \backslash S$ which approaches $+\infty$ continuously on $S$, then $L$-cap $S=0$; and that if there exists a bounded positive $L$-subsolution on $D \backslash S$ which approaches 0 continuously on $\partial D$, then $L$-cap $S>0$.

We recall that for the Laplacian, a set has positive capacity if it has positive $h$-Hausdorff measure for some $h>0$ satisfying $\int_{0}^{1} h(r) / r^{n-1} d r<\infty$; and a set has zero capacity if it has finite $(n-2)$-dimensional Hausdorff measure when $n \geq 3$, or finite logarithmic measure when $n=2$. Therefore $n-2$ is the critical dimension for studying sets of capacity zero.

We shall prove the following:

Theorem 1. Let $n \geq 2$ and $n-2<\alpha<n$. Then there exist a constant $\Lambda_{n, \alpha}>1$, a compact set $S \subseteq D$ of Hausdorff dimension $\alpha$, an operator $L=$ $\sum a_{i j} \partial^{2} / \partial x_{i} \partial x_{j}$ with coefficients bounded smooth in $D \backslash S$, satisfying

$$
|\xi|^{2} \leq \sum a_{i j}(x) \xi_{i} \xi_{j} \leq \Lambda_{n, \alpha}|\xi|^{2}, \quad x, \xi \in \mathbb{R}^{n},
$$

so that $S$ has zero $L$-capacity in the sense $(0.1)$. In fact, there is a positive $L$ supersolution $v(\not \equiv+\infty)$ in $D \backslash S$ approaching $+\infty$ continuously on $S$. Moreover,

$$
\Lambda_{n, \alpha}=1+O(1)(\alpha-n+2) \quad \text { as } \alpha \rightarrow n-2,
$$

and

$$
\Lambda_{n, \alpha}=O(1)(n-\alpha)^{-1} \quad \text { as } \alpha \rightarrow n,
$$

with the $O(1)$ terms positive and independent of $n$ and $\alpha$.

We believe that $(0.3)$ is sharp, and do not know whether $(0.4)$ can be improved.

Theorem 2. Let $n \geq 2, a>0$, and $h(r)=r^{n-2}\left(\log \frac{1}{r}\right)^{-1-a}$. Then there exist $a$ constant $\beta>0$, a compact set $S \subseteq D$ of dimension $n-2$, positive Hausdorff $h$-measure, an operator $L=\sum a_{i j} \partial^{2} / \partial x_{i} \partial x_{j}$ with coefficients continuous in $\mathbb{R}^{n}$, smooth off $S$ satisfying

$$
|\xi|^{2} \leq \sum a_{i j}(x) \xi_{i} \xi_{j} \leq\left\{1+\beta\left(\log \frac{1}{\operatorname{dist}(x, S)}\right)^{-1}\right\}|\xi|^{2}
$$


for all $x, \xi \in \mathbb{R}^{n}$, and a positive $L$-supersolution $v$ on $D$, which is an $L$ solution on $D \backslash S$ and approaches $+\infty$ continuously on $S$. In particular, $S$ has zero L-capacity, and positive capacity for the Laplacian.

Theorem 3. Let $n \geq 3, a>0$, and $h(r)=r^{n-2}\left(\log \frac{1}{r}\right)^{a}$. Then there exist a compact set $S \subseteq D$ of dimension $n-2$, vanishing $h$-measure, a constant $\beta>0$, an operator $L=\sum a_{i j} \partial^{2} / \partial x_{i} \partial x_{j}$ with coefficients continuous in $\mathbb{R}^{n}$, smooth off $S$ and satisfying

$$
\left\{1-\beta\left(\log \frac{1}{\operatorname{dist}(x, S)}\right)^{-1}\right\}|\xi|^{2} \leq \sum a_{i j}(x) \xi_{i} \xi_{j} \leq|\xi|^{2}
$$

for $x, \xi \in \mathbb{R}^{n}$, and a bounded positive L-supersolution $w$ on $D$, which is an $L$-solution on $D \backslash S$. Thus $S$ has zero capacity for the Laplacian, and positive capacity for the operator $L$.

Theorem 4. Let $n \geq 3$ and $0<\alpha<n-2$. Then there exist a positive constant $\lambda_{n, \alpha}<1$, a compact set $S \subseteq D$ of dimension $\alpha$, an operator $L=$ $\sum a_{i j} \partial^{2} / \partial x_{i} \partial x_{j}$ with $a_{i j}$ bounded smooth off $S$, satisfying

$$
\lambda_{n, \alpha}|\xi|^{2} \leq \sum a_{i j}(x) \xi_{i} \xi_{j} \leq|\xi|^{2}, \quad x, \xi \in \mathbb{R}^{n},
$$

so that $S$ has positive $L$-capacity. In fact, there exists a bounded positive $L$ subsolution $w$ on $D \backslash S$ which vanishes continuously on $\partial D$. Moreover

$$
\lambda_{n, \alpha}=(1-2 \alpha)(n-1)^{-1}, \quad 0<\alpha<1 / 4,
$$

and

$$
\lambda_{n, \alpha}=1-O(1)(n-2-\alpha) \text { as } \alpha \rightarrow n-2,
$$

with the $O(1)$ term positive and independent of $n$ and $\alpha$.

Since it is known that a point can have positive $L$-capacity, the only new part of Theorem 4 is the relation between the ellipticity constants and the dimension.

In the proofs of all four theorems, we start with the Laplace operator, then modify the coefficients on a sequence of rings, accumulating on a Cantor set $S$, so that on the rings all eigenvalues are greater than 1 (or less than 1). When all are chosen properly, it will produce an $L$-supersolution which grows faster than (or slower than) the fundamental solution of the Laplacian near each point in $S$. This explains the relation between the normalization of the ellipticity constants and the size of the set $S$.

A related subject, the boundary regularity problem for the operator $L$, has been studied by many. A partial list includes [4, 7, 8, 9. 10, 11, 12, and 13].

\section{Preliminary lemmas}

Let $\Delta$ be the Laplace's operator and $r=|x|$ for $x \in \mathbb{R}^{n}$.

Lemma 1. Let $B(x)$ be positive continuous in a domain $\Omega$, and let

$$
L=\frac{B(x)}{n-1} \sum \frac{\partial^{2}}{\partial x_{i}^{2}}-\left(\frac{B(x)}{n-1}-1\right) \sum \frac{x_{i} x_{j}}{|x|^{2}} \frac{\partial^{2}}{\partial x_{i} \partial x_{j}}
$$


on $\Omega \backslash\{0\}$. Then the coefficients $a_{i j}$ of $L$ are continuous, symmetric on $\Omega \backslash\{0\}$, satisfying

$$
|\xi|^{2} \leq \sum a_{i j}(x) \xi_{i} \xi_{j} \leq \frac{B(x)}{n-1}|\xi|^{2} \quad \text { when } B(x) \geq n-1,
$$

and

$$
\frac{B(x)}{n-1}|\xi|^{2} \leq \sum a_{i j}(x) \xi_{i} \xi_{j} \leq|\xi|^{2} \text { when } B(x) \leq n-1 .
$$

The coefficients of $L$ can be extended to be continuous on $\Omega$ if $B(0)=n-1$. Moreover, $|x|^{-B+1}$ is a solution of $L=O(x \neq 0)$, when $B(x) \equiv a$ constant $B$.

The characteristic values of $\left(a_{i j}(x)\right)$ are $1, B(x) /(n-1), B(x) /(n-1), \ldots$, $B(x) /(n-1)$; and for $x \neq 0$,

$$
L=\frac{B(x)}{n-1} \Delta-\left(\frac{B(x)}{n-1}-1\right) \frac{\partial^{2}}{\partial r^{2}}=\frac{\partial^{2}}{\partial r^{2}}+\frac{B(x)}{r} \frac{\partial}{\partial r}+\frac{B(x)}{n-1} r^{-2} \delta,
$$

where $\delta$ is the Beltrami operator in the spherical coordinates. Whence the lemma follows.

Denote by $D(x, a)$ the closed ball centered at $x$ of radius $a$, and recall that $D=D(0,1)$. When $U$ is a ball, denote by $c U$ the ball concentric to $U$ of radius $c$ times that of $U$.

Lemma 2. Let $0<\delta<\frac{1}{16}$ and $D(a, r) \subseteq D(0, \delta)$. Then there exists a diffeomorphism $y=T x$ from $\mathbb{R}^{n}$ onto $\mathbb{R}^{n}$, which fixes every point in $\mathbb{R}^{n} \backslash D\left(0, \frac{9}{16}\right)$, maps each point $x$ in $D(a, r)$ to $x-a$, and satisfies on $D\left(0, \frac{9}{16}\right)$ :

$$
\begin{gathered}
\frac{\partial y_{i}}{\partial x_{j}}-\delta_{i j}=c(x) a_{i}\left(x_{j}-a_{j}\right), \\
\left|\sum_{i} \frac{\partial^{2} y_{i}}{\partial x_{l} \partial x_{m}} \xi_{i}\right| \leq 272 \delta|\xi|,
\end{gathered}
$$

where $0<c(x)<32, \delta_{i j}=1$ when $i=j$ and $\delta_{i j}=0$ when $i \neq j$. Moreover if $\left(a_{i j}(x)\right)$ is symmetric positive definite with all its eigenvalues bounded above by $\Lambda$, and

$$
b_{i j}(x)=\sum_{l, m} a_{l m}(x) \frac{\partial y_{i}}{\partial x_{l}} \frac{\partial y_{j}}{\partial x_{m}},
$$

then

$$
\left|\sum_{i, j} b_{i j} \xi_{i} \xi_{j}-\sum_{i, j} a_{i j} \xi_{i} \xi_{j}\right| \leq 128 \delta \Lambda|\xi|^{2}
$$

and

$$
\left|\sum b_{i i}-\sum a_{i i}\right| \leq 128 \delta \Lambda \text {. }
$$

Proof. Let

$$
\psi(s)= \begin{cases}10 s^{3}-15 s^{4}+6 s^{5}, & 0 \leq s \leq 1, \\ 0, & s<0 \\ 1, & s>1 ;\end{cases}
$$


and note that $\psi$ is $C^{2}, 0 \leq \psi \leq 1,0 \leq \psi^{\prime} \leq 15 / 8$ and $\left|\psi^{\prime \prime}\right| \leq 10 / \sqrt{3}$. Let

$$
\varphi(t)=1-\psi\left(\frac{t-\delta^{2}}{\frac{1}{4}-\delta^{2}}\right) .
$$

Thus $\varphi=0$ for $t \geq \frac{1}{4}, \varphi=1$ for $t \leq \delta^{2}, 0 \leq \varphi \leq 1,-8 \leq \varphi^{\prime} \leq 0$ and $\left|\varphi^{\prime \prime}\right|<160$. Then $T x=x-\varphi\left(|x-a|^{2}\right) a$ is a diffeomorphism on $\mathbb{R}^{m}$ that fixes every point in $\mathbb{R}^{m} \backslash D\left(0, \frac{9}{16}\right)$ and maps $x \in D(a, r)$ to $x-a$. Moreover, on $D\left(0, \frac{9}{16}\right), T$ satisfies $(1.3)$ and (1.4) with $c(x)=-2 \varphi^{\prime}\left(|x-a|^{2}\right)$.

To show (1.5), we let $x \in D\left(0, \frac{9}{16}\right)$, and note that

$$
\begin{aligned}
b_{i j}-a_{i j}= & \sum_{l, m} a_{l m}\left[\delta_{i l}+c(x) a_{i}\left(x_{l}-a_{l}\right)\right]\left[\delta_{j m}+c(x) a_{j}\left(x_{m}-a_{m}\right)\right]-a_{i j} \\
= & c(x) \sum_{m} a_{i m} a_{j}\left(x_{m}-a_{m}\right)+c(x) \sum_{l} a_{l j} a_{i}\left(x_{l}-a_{l}\right) \\
& +c(x)^{2} \sum_{l, m} a_{l m} a_{i} a_{j}\left(x_{l}-a_{l}\right)\left(x_{m}-a_{m}\right) .
\end{aligned}
$$

Thus

$$
\begin{aligned}
& \left|\sum_{i, j} b_{i j} \xi_{i} \xi_{j}-\sum_{i, j} a_{i j} \xi_{i} \xi_{j}\right| \\
& \leq c(x)\left|\sum_{j} \sum_{i, m} a_{i m} \xi_{i}\left(x_{m}-a_{m}\right) a_{j} \xi_{j}\right|+c(x)\left|\sum_{i} \sum_{j, l} a_{l j} \xi_{j}\left(x_{l}-a_{l}\right) a_{i} \xi_{i}\right| \\
& \quad+c(x)^{2}\left|\sum_{i, j} \sum_{l, m} a_{l m}\left(x_{l}-a_{l}\right)\left(x_{m}-a_{m}\right) a_{i} a_{j} \xi_{i} \xi_{j}\right| .
\end{aligned}
$$

Since $|a|<\delta,|x-a|<1$ and eigenvalues of $\left(a_{i j}\right)$ are bounded above by $\Lambda$, we conclude that

$$
\left|\sum b_{i j} \xi_{i} \xi_{j}-\sum a_{i j} \xi_{i} \xi_{j}\right| \leq 2 c(x) \Lambda \delta+c(x)^{2} \Lambda \delta^{2} \leq 128 \Lambda \delta .
$$

Similarly,

$$
\begin{aligned}
& \left|\sum_{i} b_{i i}-\sum a_{i i}\right| \leq c(x)\left|\sum_{i, m} a_{i m} a_{i}\left(x_{m}-a_{m}\right)\right|+c(x)\left|\sum_{i, l} a_{l i} a_{i}\left(x_{l}-a_{l}\right)\right| \\
& +c(x)^{2}\left|\sum_{i} a_{i}^{2} \sum_{l, m} a_{l m}\left(x_{l}-a_{l}\right)\left(x_{m}-a_{m}\right)\right| \leq 128 \Lambda \delta .
\end{aligned}
$$

\section{THE CONSTRUCTION}

Given $B^{*} \geq n-1$, integer $k_{0}>0$, let $\left\{\delta_{k}\right\},\left\{r_{k}\right\}$, and $\left\{N_{k}\right\}$ be sequences satisfying $0<\delta_{k}<\left(2400 B^{*}\right)^{-1}, 0<r_{k}<r_{k-1}<r_{1} \leq \frac{1}{2}$ and $16 \sqrt{n} / \delta_{k}<N_{k}<$ $r_{k-1} / r_{k}$, for $k \geq k_{0}$. Then $r_{k+1}<\delta_{k+1} N_{k+1} r_{k+1}<N_{k+1} r_{k+1}<r_{k}$ for $k \geq k_{0}$. Let [ ] be the greatest integer function, $I_{k_{0}}=1$ and $I_{k}=\prod_{j=k_{0}}^{k}\left[\delta_{j} N_{j} / 16 \sqrt{n}\right]^{n}$ for $k>k_{0}$. 
Denote by $D_{k_{0}, 1}=D\left(0, r_{k_{0}}\right)$. After $\left\{D_{k, l}: 1 \leq l \leq I_{k}\right\}$ are selected for some $k \geq k_{0}$ we let $\mathscr{D}_{k, l}$ be the ball $\delta_{k+1} N_{k+1} r_{k+1} r_{k}^{-1} D_{k, l}$ of radius $\delta_{k+1} N_{k+1} r_{k+1}$; and choose from each $\mathscr{D}_{k, l}$ a number of $\left[\delta_{k+1} N_{k+1} / 16 \sqrt{n}\right]^{n}$ balls of radius $r_{k+1}$ to form the collection $\left\{D_{k+1, l}: 1 \leq l \leq I_{k+1}\right\}$. Moreover, we require their doublings $\left\{2 D_{k+1, l}\right\}$ to be mutually disjoint and contained in $\bigcup_{l} \mathscr{D}_{k, l}$. Let $S$ be the Cantor set defined by $S=\bigcap_{k=k_{0}}^{\infty}\left(\bigcup_{l=1}^{I_{k}} D_{k, l}\right)$. And let $\mu$ be the continuous measure on $S$, defined by $\mu\left(D_{k, l}\right)=I_{k}^{-1}$ for all $k \geq k_{0}$ and $1 \leq l \leq I_{k}$. For $k \geq k_{0}$, denote by $P_{k, l}$ the center of $D_{k, l}$,

$$
R_{k, l}=\left\{N_{k+1} r_{k+1} \leq\left|x-P_{k, l}\right| \leq r_{k}\right\} \text {, }
$$

and

$$
R_{k, l}^{\prime}=\left\{\frac{3}{4} N_{k+1} r_{k+1} \leq\left|x-P_{k, l}\right| \leq \frac{5}{4} r_{k}\right\},
$$

and note that $\left\{R_{k, l}^{\prime}: k \geq k_{0}, 1 \leq l \leq I_{k}\right\}$ are mutually disjoint.

Let $B(r)$ be a smooth function for $r>0$, satisfying $n-1 \leq B(r) \leq B^{*}$, with

$$
\begin{gathered}
B(r) \equiv n-1 \quad \text { on }\left\{r>\frac{5}{4} r_{k_{0}}\right\} \cup \bigcup_{k=k_{0}}^{\infty}\left[\frac{5}{4} r_{k}, \frac{3}{4} N_{k} r_{k}\right], \\
B(r)>n-1 \text { on } \bigcup_{k \geq k_{0}}\left[N_{k} r_{k}, r_{k-1}\right],
\end{gathered}
$$

and $B(r)$ monotone in each of the remaining intervals. Define on $\mathbb{R}^{n}$ an elliptic operator

$$
L= \begin{cases}\Delta, & \text { on } \mathbb{R}^{n} \backslash \bigcup_{k, l} R_{k, l}^{\prime}, \\ \frac{B(r)}{n-1} \Delta-\left(\frac{B(r)}{n-1}-1\right) \frac{\partial^{2}}{\partial r^{2}}, & \text { at } x+P_{k, l} \in R_{k, l}^{\prime},\end{cases}
$$

where $r=|x|$. Rewrite $L$ in the standard form $\sum a_{i j} \partial^{2} / \partial x_{i} \partial x_{j}$. We note from Lemma 1 and properties of $B(r)$ that the coefficients $a_{i j}$ are symmetric and are smooth off $S$; and that $a_{i j}$ are continuous on $\mathbb{R}^{n}$ if $\lim _{r \rightarrow 0} B(r)=$ $n-1$. Let

$$
B_{k}=\sup \left\{B(r): 0<r \leq N_{k} r_{k}\right\},
$$

from (1.1) it follows that

$$
|\xi|^{2} \leq \sum a_{i j} \xi_{i} \xi_{j} \leq \frac{B_{k}}{n-1}|\xi|^{2} \quad \text { on } R_{k, l}^{\prime}
$$

Next, we construct positive $L$-supersolutions.

Fix a point $x_{0} \in S$ and rearrange the indices if necessary, we may assume that $x_{0} \in \bigcap_{k} D_{k, 1}$. Let

$$
\begin{aligned}
& D_{k, 1}^{\prime}=\left\{\left|x-P_{k, 1}\right| \leq \frac{5}{4} r_{k}\right\}, \\
& D_{k, 1}^{\prime \prime}=\left\{\left|x-P_{k, 1}\right| \leq \frac{3}{4} N_{k+1} r_{k+1}\right\}, \\
& S_{k, 1}=D_{k-1,1}^{\prime \prime} \backslash D_{k, 1}^{\prime} ;
\end{aligned}
$$


and note that $D_{k, 1}^{\prime \prime} \subseteq D_{k, 1} \subseteq D_{k, 1}^{\prime} \subseteq D_{k-1,1}^{\prime \prime}$ and $D_{k, 1}^{\prime} \subseteq D\left(P_{k-1,1}, \delta_{k} N_{k} r_{k}\right)$. Observe also that

$$
\mathbb{R}^{n} \backslash\left\{x_{0}\right\}=\bigcup_{k \geq k_{0}} R_{k, 1}^{\prime} \cup \bigcup_{k \geq k_{0}+1} S_{k, 1} \cup\left\{|x| \geq \frac{5}{4} r_{k_{0}}\right\} ;
$$

and that $\bigcup_{k} R_{k, 1}^{\prime}$ and $\bigcup_{k} S_{k, 1}$ meet on the boundaries only. Denote by

$$
\begin{aligned}
& a^{k}=\left(a_{1}^{k}, a_{2}^{k}, \ldots, a_{n}^{k}\right)=P_{k, 1}-P_{k-1,1}, \\
& x^{k}=\left(x_{1}^{k}, x_{2}^{k}, \ldots, x_{n}^{k}\right)=x-P_{k-1,1},
\end{aligned}
$$

then $\left|a^{k}\right|<\delta_{k} N_{k} r_{k}$ and $\left|x^{k}-a^{k}\right| \leq N_{k} r_{k}$ if $x^{k} \in S_{k, 1}$.

Applying Lemma 2 to $D_{k-1,1}^{\prime \prime}$ and $D_{k, 1}^{\prime}$ instead of $D\left(0, \frac{3}{4}\right)$ and $D(a, r)$ for each $k \geq 1+k_{0}$ in succession, we obtain, after a scale change, a diffeomorphism $T$ from $\mathbb{R}^{n} \backslash\left\{x_{0}\right\}$ onto $\mathbb{R}^{n} \backslash\{0\}$ so that $T$ fixes every point in $\left\{|x|>\frac{3}{4} N_{k_{0}+1} r_{k_{0}+1}\right\}$, and is a translation on $R_{k, 1}^{\prime}$ for each $k \geq 1+k_{0}$ with

$$
T\left(R_{k, 1}^{\prime}\right)=\left\{\frac{3}{4} N_{k+1} r_{k+1} \leq|y| \leq \frac{5}{4} r_{k}\right\}
$$

and that for $x \in S_{k, 1}$,

$$
\left|\frac{\partial y_{i}}{\partial x_{j}}-\delta_{i j}\right| \leq 32\left|a_{i}^{k}\right|\left|x_{j}^{k}-a_{j}^{k}\right|\left(N_{k} r_{k}\right)^{-2} \leq 32 \delta_{k},
$$

$$
\left|\sum_{j} \frac{\partial^{2} y_{j}}{\partial x_{l} \partial x_{m}} \xi_{j}\right| \leq 272 \delta_{k}|\xi|\left(N_{k} r_{k}\right)^{-1}
$$

and

$$
T\left(S_{k, 1}\right)=\left\{\frac{5}{4} r_{k}<|y| \leq \frac{3}{4} N_{k} r_{k}\right\} .
$$

Let $T\left(x_{0}\right)=0$ and note that $T$ is homeomorphic on $\mathbb{R}^{n}$.

Let $M$ be the operator on $\mathbb{R}^{n} \backslash\{0\}$ defined by $M v(y) \equiv L(v \circ T)(x)$ when $y=T x$; that is,

$$
M=\sum_{i, j} b_{i j} \frac{\partial^{2}}{\partial y_{i} \partial y_{j}}+\sum_{j} b_{j} \frac{\partial}{\partial y_{j}}
$$

with

$$
b_{i j}=\sum_{l, m} a_{l m} \frac{\partial y_{i}}{\partial x_{l}} \frac{\partial y_{j}}{\partial x_{m}} \quad \text { and } \quad b_{j}=\sum_{l, m} a_{l m} \frac{\partial^{2} y_{j}}{\partial x_{l} \partial x_{m}} .
$$

Thus $M$ is the Laplacian on $\left\{|y| \geq \frac{5}{4} r_{k_{0}}\right\}$. Since $T$ is a translation on $R_{k, 1}^{\prime}$,

$$
M=\frac{B(\rho)}{n-1} \Delta-\left(\frac{B(\rho)}{n-1}-1\right) \frac{\partial^{2}}{\partial \rho^{2}} \quad \text { on } T\left(R_{k, 1}^{\prime}\right)
$$

where $\rho=|y|$. In view of (1.5) and (2.5), we obtain after a scale change that for $x \in S_{k, 1}$,

$$
\begin{aligned}
& \left|\sum b_{i j}(T x) \xi_{i} \xi_{j}-\sum a_{i j}(x) \xi_{i} \xi_{j}\right| \\
& \leq 128 \delta_{k} \sup _{S_{k, 1}} \sum a_{i j}(x) \xi_{i} \xi_{j} \leq \frac{128}{n-1} B_{k} \delta_{k}|\xi|^{2}
\end{aligned}
$$


The last inequality follows from (2.3) and the fact that $S_{k, 1}$ contains rings from $\left\{R_{k, l}^{\prime}\right\}_{l}$ but none from the larger ones $\left\{R_{k-1, l}^{\prime}\right\}_{l}$. Similarly it follows from (1.6) and (2.5) that

$$
\left|\sum b_{i i}(T x)-\sum a_{i i}(x)\right| \leq \frac{128}{n-1} B_{k} \delta_{k} \quad \text { on } S_{k, 1} .
$$

Let $f$ be a smooth function on $r>0$, bounded above by $B(r)$, with values $f(r) \equiv 0$ for $r \geq \frac{5}{4} r_{k_{0}}, f(r)=B(r)$ on $\bigcup_{k \geq k_{0}}\left[N_{k+1} r_{k+1}, r_{k}\right]$,

$$
f(r)=\left(n-2+\frac{n-1}{B_{k}}\right)\left(1-1200 B^{*} \delta_{k}\right) \text { on }\left[\frac{5}{4} r_{k}, \frac{3}{4} N_{k} r_{k}\right] \text {, }
$$

for each $k \geq 1+k_{0}$, and monotone in each of the remaining intervals. Define for $\rho=|y|<1$,

$$
u(y) \equiv u(\rho) \equiv \int_{\rho}^{1} \exp \int_{t}^{1} \frac{f(s)}{s} d s d t
$$

and claim that

$$
M u \leq 0 \text { in } D \backslash\{0\} .
$$

The idea of defining a radial $M$-supersolution in the form (2.11) comes from Gilbarg and Serrin [5] and Bauman [4]. It follows from (2.7) and the fact that $f(r) \leq B(r)$ that $M u \leq 0$ on $\left\{\frac{5}{4} r_{k_{0}}<|y|<1\right\} \cup \bigcup_{k} T\left(R_{k, 1}^{\prime}\right)$. On $T\left(S_{k, 1}\right)$, we note that

$$
M u(y)=\frac{u^{\prime}(\rho)}{\rho}\left[-\sum_{i, j} b_{i j} \frac{y_{i} y_{j}}{\rho^{2}} f(\rho)+\sum_{i} b_{i i}-\sum_{i, j} b_{i j} \frac{y_{i} y_{j}}{\rho^{2}}+\sum_{j} b_{j} y_{j}\right] .
$$

Eigenvalues of $\left(a_{i j}(x)\right)$ are in the form $1, \Lambda(x), \Lambda(x), \ldots, \Lambda(x)$, with

$$
1 \leq \Lambda(x) \leq \frac{B_{k}}{n-1} \quad \text { on } S_{k, 1} .
$$

We obtain from (2.6) that

$$
\left|\sum b_{j} y_{j}\right|=\left|\sum_{l, m} a_{l m} \sum_{j} \frac{\partial^{2} y_{j}}{\partial x_{l} \partial x_{m}} y_{j}\right| \leq 272 \delta_{k} B_{k} \frac{n}{n-1}
$$

on $T\left(S_{k, 1}\right)$. For $x \in S_{k, 1}, y=T x$, and $\rho=|y|$, we obtain from (2.8), (2.9), (2.10), (2.13) and the assumptions $B(r) \leq B^{*}$ and $\delta_{k}<\left(2400 B^{*}\right)^{-1}$ that

$$
\begin{gathered}
\frac{\sum b_{i i}+\sum b_{j} y_{j}}{\sum b_{i j} \frac{y_{i} y_{j}}{\rho^{2}}}-1 \geq \frac{\sum a_{i i}-\frac{128}{n-1} B_{k} \delta_{k}-272 \frac{n}{n-1} B_{k} \delta_{k}}{\sum a_{i j} \frac{y_{i} y_{j}}{\rho^{2}}+\frac{128}{n-1} B_{k} \delta_{k}}-1 \\
\quad \geq \frac{1+(n-1) \Lambda(x)-\left(\frac{128}{n-1}+272 \frac{n}{n-1}\right) B_{k} \delta_{k}}{\Lambda(x)+\frac{128}{n-1} B_{k} \delta_{k}}-1 \\
\geq\left(n-2+\frac{n-1}{B_{k}}\right)\left(1-1200 B^{*} \delta_{k}\right) \geq f(|y|) .
\end{gathered}
$$

Hence $M u \leq 0$ on $T\left(S_{k, 1}\right)$, and (2.12) is proved. 
Let $H_{x_{0}}\left(x_{0}\right)=+\infty$ and

$$
H_{x_{0}}(x)=u(T x) \quad \text { on } D \backslash\left\{x_{0}\right\} .
$$

Since $L H_{x_{0}} \leq 0$ on $D \backslash\left\{x_{0}\right\}$ and the coefficients of $L$ are smooth off $S, H_{x_{0}}$ is an $L$-supersolution in $D \backslash S$. We shall estimate the growth of $H_{x_{0}}(x)$ near $x_{0}$.

In the rest of the paper, $C$ denotes positive constants depending at most on $n, \alpha$ and $a$ in the theorems; its value may vary from line to line.

\section{Proof OF THEOREM 1}

Let

$$
B=\frac{2(n-1)+2 n(\alpha+2-n)}{n-\alpha},
$$

and note that $B>\alpha+1>n-1, B \rightarrow n-1$ as $\alpha \rightarrow n-2$, and

$$
\frac{\alpha}{n}<\frac{B-(1+\alpha)}{B-(n-2)-(n-1) / B}<1 \text {. }
$$

Choose $\alpha^{\prime}, \alpha<\alpha^{\prime}<n$, so that

$$
\frac{\alpha}{n}<\frac{B-\left(1+\alpha^{\prime}\right)}{B-(n-2)-(n-1) / B}<1,
$$

and denote by

$$
A=\frac{B-\left(1+\alpha^{\prime}\right)}{B-(n-2)-(n-1) / B} \quad \text { and } \quad E=A-\frac{\alpha}{n} .
$$

Let

$$
\delta_{k}=\frac{16 \sqrt{n}}{k}, \quad N_{k}=k^{A / E} \quad \text { and } \quad r_{k}=(k !)^{-1 / E}
$$

and note that $N_{k} \leq r_{k-1} / r_{k}$ for $k \geq 1$. To specify $B(r)$ in (2.1), we let

$$
B(r) \equiv B \quad \text { on } \bigcup_{k \geq k_{0}}\left[N_{k+1} r_{k+1}, r_{k}\right]
$$

and note that $n-1 \leq B(r) \leq B$. Choose and fix integer $k_{0} \geq 10^{5} B \sqrt{n}$.

It is clear that

$$
\lim _{k \rightarrow \infty}(k !)^{\alpha / E}\left(\delta_{k} N_{k} r_{k}\right)^{\gamma}=0 \text { if } \gamma>\alpha
$$

and that

$$
\lim _{k \rightarrow \infty}\left(2^{-k}(k-1) !\right)^{-\alpha / E} /\left(\delta_{k} N_{k} r_{k}\right)^{\eta}=0, \quad \text { if } \eta<\alpha .
$$

Note that there are $I_{k}=\prod_{j=k_{0}}^{k}\left[j^{A / E-1}\right]^{n}$ balls in $\left\{D_{k, l}\right\}_{l}$ and that

$$
\left(\frac{k !}{2^{k} k_{0} !}\right)^{\alpha / E} \leq I_{k} \leq k !^{\alpha / E}
$$

when $k$ is large. And recall that $\mu$ is the continuous measure on $S$ defined by

$$
\mu\left(D_{k, l}\right)=I_{k}^{-1} \text { for each } l \text { and } k \geq k_{0} .
$$


The smallest balls that carry a $\mu$-measure $I_{k-1}^{-1}$ have radii proportional to $\delta_{k} N_{k} r_{k}$. Using (3.5) and (3.6) one may check that for each $\eta<\alpha, \mu(D(x, r)) \leq$ $C_{\eta} r^{\eta}$ for all $x \in \mathbb{R}^{n}$ and $r>0$. Hence $S$ has positive $\eta$-dimensional measure for all $\eta<\alpha$. In view of (3.4), $S$ has Hausdorff dimension $\alpha$.

For $0<t<r_{k_{0}}$, let $K \equiv K(t)$ be the largest integer so that $r_{K} \geq t$. We deduce from (2.10), (2.11), and (3.3) that, for $0<\rho<r_{k_{0}}$,

$$
\begin{aligned}
u(\rho) \geq \int_{\rho}^{r_{K_{0}}} \exp \{ & \int_{t}^{r_{K_{0}}} \frac{B}{S} d s \\
& \left.\quad-\sum_{k=k_{0}}^{K} \int_{r_{k}}^{N_{k} r_{k}} B-\left(n-2+\frac{n-1}{B}\right)\left(1-1200 B \delta_{k}\right) \frac{d s}{s}\right\} d t .
\end{aligned}
$$

And note from the choices of $A$ and $\delta_{k}$ that

$$
\begin{aligned}
\sum_{k=k_{0}}^{K} & \int_{r_{k}}^{N_{k} r_{k}} B-\left(n-2+\frac{n-1}{B}\right)\left(1-1200 B \delta_{k}\right) \frac{d s}{s} \\
& \leq \sum_{k=k_{0}}^{K}\left[\left(B-n+2-\frac{n-1}{B}\right)+1200 B \delta_{k}(n-1)\right] \frac{A}{E} \log k \\
& \leq \sum_{k=k_{0}}^{K} \frac{\left(B-\alpha^{\prime}-1\right)}{E} \log k+C \frac{\log k}{k} \\
& =C+\left(B-\alpha^{\prime}-1\right) \log \frac{1}{t}+C\left(\log \log \frac{1}{t}\right)^{2} .
\end{aligned}
$$

Therefore, for $0<\rho<r_{k_{0}}$,

$$
u(\rho) \geq \int_{\rho}^{r_{k_{0}}} \exp \left\{-C+\left(\alpha^{\prime}+1\right) \log \frac{1}{t}-C\left(\log \log \frac{1}{t}\right)^{2}\right\} d t \geq C \rho^{-\gamma}
$$

for some $\gamma$ satisfying $\alpha<\gamma<\alpha^{\prime}$. From the property (2.5) of the transformation $T$, it follows that

$$
H_{x_{0}}(x) \geq C\left|x-x_{0}\right|^{-\gamma} \text { when }|x|<r_{k_{0}} .
$$

Let $v(x)=\int_{S} H_{z}(x) d \mu(z)$, where $\mu$ is the measure defined in (3.7). Clearly $v$ is an $L$-supersolution on $D \backslash S$. In view of (3.4), (3.6), and (3.8), $v$ approaches $+\infty$ as $x \rightarrow x_{0}$ for every $x_{0} \in S$. Since $f(r)$ is bounded, $v<+\infty$ on $D \backslash S$.

Clearly (0.2) holds with $\Lambda_{n, \alpha}=B /(n-1)$.

The number $B$ was chosen so that among other properties, (3.1) holds. As a consequence, $(0.3)$ and $(0.4)$ follow.

\section{Proof of Theorem $2(n>3)$}

Let

$$
\delta_{k}=\frac{16 \sqrt{n}}{k^{3 / 2}}, \quad N_{k}=k^{2 n-5 / 2}
$$

and

$$
r_{k}=(k !)^{-2 n+(4+(2+a) /(n-2)) / k}
$$


for $k \geq 1$. Choose an integer $k_{0} \geq 10^{5}(1+a) n$, so that $N_{k} \leq r_{k-1} / r_{k}$ for $k \geq k_{0}$. It is easy to check that

$$
\begin{gathered}
\lim _{k \rightarrow \infty}(k !)^{2 n(n-2)} r_{k}^{\gamma}=0 \quad \text { if } \gamma>n-2, \\
\lim _{k \rightarrow \infty}(k-1) !^{-2 n(n-2)} /\left(\delta_{k} N_{k} r_{k}\right)^{n-2}\left(\log \frac{1}{\delta_{k} N_{k} r_{k}}\right)^{-1-a}=0, \\
\lim _{k \rightarrow \infty}(k-1) !^{-2 n(n-2)} /\left(\delta_{k} N_{k} r_{k}\right)^{n-2}\left(\log \frac{1}{\delta_{k} N_{k} r_{k}}\right)^{-3-a}=\infty
\end{gathered}
$$

There are $\left(k ! / k_{0} !\right)^{2 n(n-2)}$ balls in $\left\{D_{k, l}\right\}_{l}$, and that

$$
\mu\left(D_{k, l}\right)=\left(k_{0} ! / k !\right)^{2 n(n-2)}
$$

for $k \geq k_{0}$. In view of (4.2) and (4.3), $S$ has Hausdorff dimension $n-2$ and positive $h$-measure, where $h(r)=r^{n-2}\left(\log \frac{1}{r}\right)^{-1-a}$.

Let

$$
\beta=12 n(1+a),
$$

and $B(r)$ be the function described in $\S 2$, with

$$
B(r)=n-1+\frac{\beta}{\log \frac{1}{r}} \text { on } \bigcup_{k \geq k_{0}}\left[N_{k+1} r_{k+1}, r_{k}\right] \text {. }
$$

Clearly $n-1 \leq B(r) \leq n-1+\beta / k_{0} \equiv B^{*}$, and $\delta_{k}<\left(2400 B^{*}\right)^{-1}$ for $k \geq k_{0}$.

Stirling's formula shows that

$$
\left(\log \frac{1}{r_{k-1}}\right)^{-1} \leq \frac{1}{2 n k \log k}\left(1+\frac{C}{\log k}\right),
$$

when $k$ is sufficiently large. Since $\left(\log \frac{1}{s}\right)^{-1}$ is an increasing function of $s$ $(0<s<1)$, and $r_{k-1} \geq N_{k} r_{k}$, we obtain from (4.7) that

$$
\begin{aligned}
1-\frac{n-1}{B_{k}} & =\frac{B_{k}-(n-1)}{B_{k}} \leq \frac{\beta}{(n-1) \log 1 / r_{k-1}} \\
& \leq \frac{\beta}{2 n(n-1) k \log k}\left(1+\frac{C}{\log k}\right)
\end{aligned}
$$

for large $k$, here $B_{k}$ is the number defined in (2.3).

Thus, for $0<\rho<r_{k_{0}}$,

$$
\begin{aligned}
u(\rho) \geq \int_{\rho}^{r_{k_{0}}} & \exp \left\{\int_{t}^{r_{k_{0}}} \frac{n-1}{S}+\frac{\beta}{s \log \frac{1}{s}} d s-\sum_{k=k_{0}}^{K} \int_{r_{k}}^{N_{k} r_{k}} \frac{\beta}{s \log \frac{1}{s}} d s\right. \\
& \left.-\sum_{k=k_{0}}^{K} \int_{r_{k}}^{N_{k} r_{k}}(n-1)-\left(n-2+\frac{n-1}{B_{k}}\right)\left(1-1200 B^{*} \delta_{k}\right) \frac{d s}{s}\right\} d t,
\end{aligned}
$$

where $K=k(t)$ is the largest integer satisfying $r_{K} \geq t$. We deduce from (4.8) 
that

$$
\begin{aligned}
& \sum_{k=k_{0}}^{K} \int_{r_{k}}^{N_{k} r_{k}}(n-1)-\left(n-2+\frac{n-1}{B_{k}}\right)\left(1-1200 B^{*} \delta_{k}\right) \frac{d s}{s} \\
& \quad \leq \sum_{k=k_{0}}^{K}\left[\frac{\beta}{2 n(n-1) k \log k}\left(1+\frac{C}{\log k}\right)+C k^{-3 / 2}\right] \log N_{k} \\
& \quad \leq C+\frac{\beta\left(2 n-\frac{5}{2}\right)}{2 n(n-1)} \log K+C \log \log K \\
& \quad \leq C+\frac{\beta\left(2 n-\frac{5}{2}\right)}{2 n(n-1)} \log \log \frac{1}{t}+C \log \log \log \frac{1}{t} .
\end{aligned}
$$

Again, from (4.7) and monotonicity of $\left(\log \frac{1}{s}\right)^{-1}$, it follows that

$$
\begin{gathered}
\sum_{k=k_{0}}^{K} \int_{r_{k}}^{N_{k} r_{k}} \frac{\beta}{s \log \frac{1}{s}} d s \leq \frac{\beta\left(2 n-\frac{5}{2}\right)}{2 n} \sum_{k=k_{0}}^{K}\left(1+\frac{C}{\log k}\right) / k \\
\leq C+\frac{\beta\left(2 n-\frac{5}{2}\right)}{2 n} \log \log \frac{1}{t}+C \log \log \log \frac{1}{t} .
\end{gathered}
$$

We conclude from (4.6), (4.9), (4.10) and (4.11) that

$$
\begin{aligned}
u(\rho) \geq \int_{\rho}^{r_{k_{0}}} \exp \{ & -C+(n-1) \log \frac{1}{t} \\
& +\beta\left[1-\frac{2 n-\frac{5}{2}}{2 n}\left(1+\frac{1}{n-1}\right)\right] \log \log \frac{1}{t}
\end{aligned}
$$

$$
\begin{aligned}
\geq C \int_{\rho}^{r_{k_{0}}} t^{-n+1}\left(\log \frac{1}{t}\right)^{\beta / 4(n-1)}\left(\log \log \frac{1}{t}\right)^{-C} d t & \left.-C \log \log \log \frac{1}{t}\right\} d t \\
\geq C \rho^{-n+2}\left(\log \frac{1}{\rho}\right)^{3+a} &
\end{aligned}
$$

for $0<\rho<r_{k_{0}}$. Therefore,

$$
H_{x_{0}}(x) \geq C\left|x-x_{0}\right|^{-n+2}\left(\log \frac{1}{\left|x-x_{0}\right|}\right)^{3+a} \text { for }|x|<r_{k_{0}}
$$

The relation

$$
\frac{2 n-\frac{5}{2}}{2 n}\left(1+\frac{1}{n-1}\right)<1
$$

used in (4.12) is prepared in the choices of $r_{k}$ and $N_{k}$.

The ellipticity of $a_{i j},(0.5)$, follows from the choice of $B(r)$; and the continuity of $a_{i j}$ in $\mathbb{R}^{n}$ follows from $\lim _{r \rightarrow 0} B(r)=n-1$. Recall that $L H_{x_{0}} \leq 0$ on $D \backslash\left\{x_{0}\right\}$; it follows from the maximum principle and the solvability of the Dirichlet problem for operators with continuous coefficients [5, pp. 220 and 252] and $H_{x_{0}}$ is $L$-supersolution in $D$.

Again, because $a_{i j}$ are continuous, Green functions $G\left(x, x_{0}\right)$ exist in $D$ (see Bauman [3, 4]). In fact, for each $x_{0} \in D, G\left(\cdot, x_{0}\right)$ is a positive $L$-solution 
in $D \backslash\left\{x_{0}\right\}$ with boundary value vanishing continuously on $|x|=1$. Let $\bar{x}=$ $\left(\frac{1}{2}, 0,0,0, \ldots 0\right)$ and assume that $G$ is normalized so that $G\left(\bar{x}, x_{0}\right)=1$. We claim that for each $x_{0} \in S$,

$$
G\left(x, x_{0}\right) \geq C\left|x-x_{0}\right|^{-n+2}\left(\log \frac{1}{\left|x-x_{0}\right|}\right)^{3+a}
$$

whenever $0<\left|x-x_{0}\right|<r_{k_{0}}$.

Let $g(r)=\sup \left\{G\left(x, x_{0}\right):\left|x-x_{0}\right|=r\right\}$ for $0<r<r_{k_{0}}$. Applying (4.13) and the maximum principle to the region $D \backslash\left\{\left|x-x_{0}\right| \leq r\right\}$, we obtain

$$
1=G\left(\bar{x}, x_{0}\right) \leq C g(r) r^{n-2}\left(\log \frac{1}{r}\right)^{-3-a} H_{x_{0}}(\bar{x}) .
$$

Because $f(r)$ is bounded and $\left|x_{0}-\bar{x}\right|>\frac{1}{4}, H_{x_{0}}(\bar{x})<C<\infty$ for all $x_{0} \in S$. Hence $g(r) \geq C r^{-n+2}\left(\log \frac{1}{r}\right)^{3+a}$ for $0<r<r_{k_{0}}$. Thus (4.14) follows from the Harnack principle.

In view of (4.14), the maximum principle and the solvability of the Dirichlet problem, $G\left(\cdot, x_{0}\right)$ is actually an $L$-supersolution on $D$. The function $v(x)=$ $\int_{S} G(x, z) d \mu(z)$ approaches $+\infty$ on $S$ in view of (4.4) and (4.14), and it is the function desired.

\section{Proof of Theorem $2(n=2)$}

Let $\delta_{k} \equiv \delta \equiv\left[50000(1+a)^{2}\right]^{-1}, N_{k} \equiv N \equiv 1600000 \sqrt{2}(1+a)^{2}, r_{k} \equiv$ $e^{-4^{k /(1+a)}} / 32 \sqrt{2}$ for $k \geq 1$. Choose integers $k_{0} \geq 1+a$ so that

$$
r_{k-1} / r_{k} \geq N^{4} \text { when } k \geq k_{0} \text {. }
$$

Note that

$$
4^{-k}\left(\log \frac{1}{N \delta r_{k}}\right)^{1+a}=1 .
$$

We note that there are $4^{k-k_{0}}$ disks in $\left\{D_{k, l}\right\}_{l}$, and that $\mu\left(D_{k, l}\right)=4^{-k+k_{0}}$. In view of (5.2), $S$ has positive finite $h$-measure for $h(r)=\left(\log \frac{1}{r}\right)^{-1-a}$.

Choose

$$
B(r)=1+20(1+a)^{2}\left(\log \frac{1}{r}\right)^{-1} \text { on } \bigcup_{k \geq k_{0}}\left[N_{k+1} r_{k+1}, r_{k}\right] .
$$

Clearly $1 \leq B(r) \leq 6(1+a)^{2}$. Let $f(r)$ be the function in $\S 2$, satisfying all the properties there except (2.10); instead, let $f(r) \equiv 0$ on $\bigcup\left[\frac{5}{4} r_{k}, \frac{3}{4} N_{k} r_{k}\right]$. The fact that $M u \leq 0$ in $D \backslash\{0\}$ is not affected by the change of $f(r)$ due to the estimate (2.14).

For $0<\rho<r_{k_{0}}$,

$$
\begin{aligned}
u(\rho) \geq \int_{\rho}^{r_{k_{0}}} \exp \left\{\int_{t}^{r_{k_{0}}}\right. & \frac{1}{s}+\frac{20(1+a)^{2}}{s \log \frac{1}{s}} d s \\
& \left.-\sum_{k=k_{0}}^{K} \int_{r_{k}}^{N_{k} r_{k}} \frac{1}{s}+\frac{20(1+a)^{2}}{s \log \frac{1}{s}} d s\right\} d t,
\end{aligned}
$$


where $K=K(t)$ is the largest integer so that $r_{K} \geq t$. We deduce from (5.1) that

$$
\sum_{k=k_{0}}^{K} \int_{r_{k}}^{N r_{k}} \frac{1}{s \log \frac{1}{s}} d s \leq C+\frac{1}{4} \int_{t}^{r_{k_{0}}} \frac{1}{s \log \frac{1}{s}} d s \leq C+\frac{1}{4} \log \log \frac{1}{t},
$$

and that

$$
\sum_{k=k_{0}}^{K} \int_{r_{k}}^{N r_{k}} \frac{1}{s} d s=\left(K-K_{0}\right) \log N \leq C+14(1+a)^{2} \log \log \frac{1}{t} .
$$

Combining the above estimates, we obtain

$$
u(\rho) \geq \int_{\rho}^{1} \exp \left\{C+\log \frac{1}{t}+(1+a)^{2} \log \log \frac{1}{t}\right\} d t \geq C\left(\log \frac{1}{\rho}\right)^{1+(1+a)^{2}}
$$

for $0<\rho<r_{k_{0}}$.

In view of (5.3), $a_{i j}$ are continuous in $D$. Thus the normalized Green function exists on $D$ and satisfies

$$
G\left(x, x_{0}\right) \geq C\left(\log \frac{1}{\left|x-x_{0}\right|}\right)^{1+(1+a)^{2}} \text { for }\left|x-x_{0}\right|<r_{k_{0}} .
$$

The function $v(x)=\int_{S} G(x, y) d \mu(y)$ has all the properties in the theorem.

\section{Proofs of Theorems 3 and 4}

We follow the constructions in $\S 2$ and indicate the necessary changes.

Given $B^{*}=n-1, k_{0},\left\{\delta_{k}\right\},\left\{r_{k}\right\}$ and $\left\{N_{k}\right\}$, let $S$ be the Cantor set and $\mu$ be the measure on $S$ defined in $\S 2$.

Let $B(r)$ be a new function, smooth on $r>0$, with values $\frac{1}{2}<B(r) \leq n-1$, satisfying (2.1),

$$
B(r)<n-1 \text { on } \bigcup_{k \geq k_{0}}\left[N_{k+1} r_{k+1}, r_{k}\right],
$$

and monotone in each of the remaining intervals. Define an operator $L$ associated with this $B(r)$ as in (2.2). Let

$$
\beta_{k}=\inf \left\{B(r): 0<r \leq N_{k} r_{k}\right\},
$$

then

$$
\frac{\beta_{k}}{n-1}|\xi|^{2} \leq \sum a_{i j} \xi_{i} \xi_{j} \leq|\xi|^{2} \quad \text { on } R_{k, l}^{\prime}
$$

Fix $x_{0} \in S$, let $y=T x$ be the diffeomorphism and $M$ be the operator defined before. Clearly $(2.5) \sim(2.7)$ are retained; and (2.8) and (2.9) can be replaced respectively by

$$
\left|\sum b_{i j}(T x) \xi_{i} \xi_{j}-\sum a_{i j}(x) \xi_{i} \xi_{j}\right| \leq 128 \delta_{k}|\xi|^{2} \text { on } S_{k, 1},
$$

and

$$
\left|\sum b_{i i}(T x) \xi_{i} \xi_{j}-\sum a_{i i}(x) \xi_{i} \xi_{j}\right| \leq 128 \delta_{k} \text { on } S_{k, 1} .
$$

Suppose that $F$ is a smooth function on $r>0$, with values $F(r) \geq B(r)$, $F(r) \equiv n-1$ for $r \geq \frac{5}{4} r_{k_{0}}, F(r)=B(r)$ on $\bigcup_{k \geq k_{0}}\left[N_{k+1} r_{k+1}, r_{k}\right]$,

$$
F(r)=\left(n-2+\frac{n-1}{\beta_{k}}\right)\left(1+5000 \delta_{k}\right) \text { on }\left[\frac{5}{4} r_{k}, \frac{3}{4} N_{k} r_{k}\right] \text {, }
$$


for each $k \geq 1+k_{0}$, and that $F$ is monotone in each of the remaining intervals. Define for $\rho=|y|<1$,

$$
U(y)=U(\rho)=\int_{\rho}^{1} \exp \int_{t}^{1} \frac{F(s)}{s} d s d t .
$$

Arguing as in $\S 2$, we conclude that for $x \in S_{k, 1}$, and $y=T x$,

$$
\frac{\sum b_{i i}+\sum b_{j} y_{j}}{\sum b_{i j} \frac{y_{i} y_{j}}{\rho^{2}}}-1 \leq f(|y|) \text {. }
$$

From this, we may deduce that $M U(y) \geq 0$ on $\{|y|<1\} \backslash\{0\}$. Thus,

$$
Q_{x_{0}}(x) \equiv U(T x) \quad \text { on } D \backslash\left\{x_{0}\right\}
$$

is an $L$-subsolution in $D \backslash S$.

To complete the proof of Theorem 3 , we let $\delta_{k}$ and $N_{k}$ be the numbers defined in (4.1), let $\tau>a /(n-2)$ and $r_{k}=(k !)^{-2 n-\tau / k}$. Fix an integer $k_{0} \geq$ $20\left(n^{2}+\tau^{2}\right)$, so that $N_{k} \leq r_{k-1} / r_{k}$ and $\delta_{k} \leq(2400 n)^{-1}$ for $k \geq k_{0}$. It is ready to check that

$$
\begin{gathered}
\lim _{k \rightarrow \infty}(k !)^{2 n(n-2)} r_{k}^{n-2}\left(\log \frac{1}{r_{k}}\right)^{a}=0, \\
\lim _{k \rightarrow \infty}((k-1) !)^{-2 n(n-2)}\left(\delta_{k} N_{k} r_{k}\right)^{-\eta}=0 \text { if } \eta<n-2,
\end{gathered}
$$

and$$
\sum_{k \geq k_{0}}((k-1) !)^{-2 n(n-2)} r_{k}^{-n+2}\left(\log \frac{1}{r_{k}}\right)^{-2 n(n+\tau)}<\infty .
$$

There are $\left(k ! / k_{0} !\right)^{2 n(n-2)}$ balls in $\left\{D_{k, l}\right\}_{l}$ for each $k \geq k_{0}$, and $\mu\left(D_{k, l}\right)=$ $\left(k_{0} ! / k !\right)^{2 n(n-2)}$. From (6.2) and (6.3) it follows that $S$ has Hausdorff dimension $n-2$, and zero $h$-measure for $h(r)=r^{n-2}\left(\log \frac{1}{r}\right)^{a}$.

Let

$$
\beta=16 n^{2}(n+\tau)
$$

and

Thus, for $0<\rho<r_{k_{0}}$,

$$
B(r)=n-1-\frac{\beta}{\log \frac{1}{r}} \text { on } \bigcup_{k \geq k_{0}}\left[N_{k+1} r_{k+1}, r_{k}\right] .
$$

$$
\begin{aligned}
U(\rho) \leq \int_{\rho}^{1} & \exp \left\{\int_{t}^{1} \frac{n-1}{s}-\frac{\beta}{s \log \frac{1}{s}} d s+C+\sum_{k=k_{0}}^{K} \int_{r_{k}}^{N_{k} r_{k}} \frac{\beta}{s \log \frac{1}{s}} d s\right. \\
& \left.+\sum_{k=k_{0}}^{K} \int_{r_{k}}^{N_{k} r_{k}}\left[-(n-1)+\left(n-2+\frac{n-1}{\beta_{k}}\right)\left(1+5000 \delta_{k}\right)\right] \frac{d s}{s}\right\} d t,
\end{aligned}
$$

where $K=K(t)$ is the largest integer satisfying $r_{K} \geq t$. Note that for large $k$, inequality (4.7) still holds and

$$
\frac{n-1}{\beta_{k}}-1 \leq \frac{9 \beta}{16 n(n-1) k \log k} \text {. }
$$

Thus $U(\rho) \leq C \rho^{-n+2}\left(\log \frac{1}{\rho}\right)^{-\beta / 8 n}$. 
Let $G\left(, x_{0}\right)$ be the normalized Green function on $D$ with

$$
G\left(\left(\frac{1}{2}, 0,0, \ldots 0\right), x_{0}\right)=1 .
$$

Arguing as in $\S 4$, we obtain

$$
G\left(x, x_{0}\right) \leq C\left|x-x_{0}\right|^{-n+2}\left(\log \frac{1}{\left|x-x_{0}\right|}\right)^{-\beta / 8 n}
$$

for all $x_{0} \in S$ and $\left|x-x_{0}\right|<r_{k_{0}}$. We may also prove that

$$
G\left(x, x_{0}\right) \rightarrow+\infty \quad \text { as } x \rightarrow x_{0}
$$

for each $x_{0} \in S$, by constructing a positive $L$-supersolution approaching $+\infty$ at $x_{0}$. Thus $G\left(\cdot, x_{0}\right)$ is an $L$-supersolution on $D$. In view of (6.4), (6.5), and (6.6),

$$
w(x)=\int_{S} G(x, y) d \mu(y)
$$

has all the properties stated in Theorem 3.

To prove Theorem 4 , we need only to verify that the coefficients of $L$ can be chosen so that $(0.6)$ and $(0.7)$ are fulfilled. Let

$$
b= \begin{cases}1-2 \alpha, & \text { if } 0<\alpha<\frac{1}{4}, \\ (n-1)\left(1-\frac{n-2-\alpha}{n-\frac{3}{2}-\alpha}\right), & \text { if } n-\frac{9}{4}<\alpha<n-2,\end{cases}
$$

and note that $\frac{1}{2}<b<1+\alpha<n-1$ and that

$$
\frac{\alpha}{n}<\frac{1+\alpha-b}{n-2+(n-1) / b-b}<1 .
$$

Choose $\alpha^{\prime}, 0<\alpha^{\prime}<\alpha$ so that

$$
\frac{\alpha}{n}<\frac{1+\alpha^{\prime}-b}{n-2+(n-1) / b-b}<1,
$$

and denote by

$$
A=\frac{1+\alpha^{\prime}-b}{n-2+(n-1) / b-b}, \quad E=A-\frac{\alpha}{n} .
$$

Let $\delta_{k}, N_{k}$ and $r_{k}$ be defined according to (3.2), associated with the current choices of $A$ and $E$; and let the function $B(r)$ in (6.1) be chosen so that

$$
B(r) \equiv b \quad \text { on } \bigcup_{k \geq k_{0}}\left[N_{k+1} r_{k+1}, r_{k}\right]
$$

It is ready to check that $S$ has dimension $\alpha$ and that the $L$-subsolution $Q_{x_{0}}(x)$ satisfies

$$
Q_{x_{0}}(x) \leq C\left|x-x_{0}\right|^{-\gamma}, \quad \text { when }\left|x-x_{0}\right|<r_{k_{0}}
$$

for some $\gamma$ with $\alpha^{\prime}<\gamma<\alpha$. The rest of the proof is routine and follows from the observation

$$
\sum_{k \geq k_{0}}(k-1) !^{-\alpha / E}\left(\delta_{k} N_{k} r_{k}\right)^{-\gamma}<\infty \quad \text { if } \gamma<\alpha .
$$




\section{REFERENCES}

1. A. Ancona, Principe de Harnack a la frontière et théorème de Fatou pour un opérateur elliptique dans un domaine Lipschitzien, Ann. Inst. Fourier (Grenoble) 28 (1978), 169-213.

2. P. Bauman, Equivalence of the Green's functions for diffusion operators in $\mathbb{R}^{n}$ : a counterexample, Proc. Amer. Math. Soc. 91 (1984), 64-68.

3. __ Positive solutions of elliptic equations in nondivergence form and their adjoints, Ark. Mat. 22 (1984), 153-173.

4. Univ. Math. J. 34 (1985), 825-844.

5. D. Gilbarg and J. Serrin, On isolated singularities of solutions of second order elliptic differential equations, J. Analyse Math. 4 (1955-56), 309-340.

6. D. Gilbarg and N. S. Trudinger, Elliptic partial differential equations of second order, 2nd ed., Springer-Verlag, 1983.

7. N. V. Krylov, The first boundary-value problem for second-order elliptic equations, Differentsial'nye Uravneniya 3 (1967), 315-326; English transl., Differential Equations 3 (1967), 158-164.

8. E. M. Landis, s-capacity and its applications to the study of solutions of a second-order elliptic equation with discontinuous coefficients, Mat. Sb. 76 (1968), 186-213; English transl., Math. USSR-Sb. 5 (1968), 177-204.

9. G. Lieberman, A generalization of the flat cone condition for regularity of solutions of elliptic equations, Proc. Amer. Math. Soc. 100 (1987), 289-294.

10. W. Littman, G. Stampacchia, and H. Weinberger, Regular points for elliptic equations with discontinuous coefficients, Ann. Scuola Norm. Sup. Pisa (3) 17 (1963), 45-79.

11. K. Miller, Exceptional boundary points for the nondivergence equation which are regular for the Laplace equation - and vice-versa, Ann. Scuola Norm. Sup. Pisa (3) 22 (1968), 315-330.

12. __ Nonequivalence of regular boundary points for the Laplace and nondivergence equations, even with continuous coefficients, Ann. Scuola Norm. Sup. Pisa (3) 24 (1970), 159-163.

13. O. N. Zograf, Example of a second-order elliptic equation with continuous coefficients for which the regularity conditions of a boundary point for the Dirichlet problem differ from the analogous conditions for the Laplace equation, Vestnik Moskov. Univ. Mat. 24 (1969), 30-39; English transl., Moscow Univ. Math. Bull. 24 (1969), 34-38.

Department of Mathematics, University of Illinois, Urbana, Illinois 61801 